\section{Erfolgreiche topische Therapie eines oberflächlichen Basalioms mit Imiquimod}

Zusammenfassung. Imiquimod ist ein topisch wirksamer Immunmodulator, der zur Therapie von Condylomata acuminata eingesetzt wird. Wir berichten über den Fall eines 73-jährigen Mannes mit einem histologisch gesicherten superfiziellen Basaliom der Schläfe, bei dem Imiquimod (Aldara ${ }^{\circledR} 5 \%$ Creme) 3-mal wöchentlich für insgesamt 12 Wochen angewendet wurde. Drei Wochen nach Behandlungsende waren weder klinisch noch histologisch Tumorreste nachweisbar (komplette Remission). Als Nebenwirkung trat eine selbstlimitierende starke lokale Entzündungsreaktion auf.

Successful Treatment of a Superficial Basal Cell Carcinoma With Topical Imiquimod. Imiquimod is an effective immune response modifier used for topical treatment of genital warts. We report on a 73-year old male patient with a biopsy-proven superfical basal cell carcinoma of the temple region. Treatment was performed by application of imiquimod $5 \%$ cream (Aldara ${ }^{\circledR}$ ) three times a week for 12 consecutive weeks. Three weeks after the end of treatment no tumour residues were detectable neither clinically nor histologically (complete remission). The cosmetic result was excellent. A strong but self-limiting inflammatory skin reaction was noted as local side effect.

\section{Einleitung}

Imiquimod (Aldara ${ }^{\circledR} 5 \%$ Creme) ist ein topisch wirksamer Immunmodulator, der zur Therapie von Condylomata acuminata eingesetzt wird [1]. Diese Substanz induziert in der Haut u.a. die Synthese von Interferonen. Die prinzipielle Wirksamkeit der lokalen Immuntherapie von Basaliomen (Basalzellkarzinomen) mit Interferonen $\alpha$ bzw. $\beta$ ist nachgewiesen $[2,3]$. Kürzlich wurde eine Pilotstudie zur topischen Anwendung von 5\% Imiquimod-Creme beim Basaliom publiziert [4]. Bei zumindest dreimal wöchentlicher Anwendung über bis zu 16 Wochen wurde bei allen so behandelten 15 Patienten eine histologisch gesicherte komplette Abheilung erzielt. Geringere Anwendungshäufigkeiten und wirkstoffreie Behandlung mit der Grundlage waren weniger wirksam bzw. unwirksam. Wir

Akt Dermatol 2001; 27: 147-149

(c) Georg Thieme Verlag Stuttgart · New York ISSN 0340-2541
L. Kowalzick, J.-M. Pönnighaus, S. Suckow

Klinik für Hautkrankheiten und Allergologie, Vogtland-Klinikum Plauen GmbH (Chefarzt: Priv.-Doz. Dr. med. habil. L. Kowalzick)

berichten über unsere Erfahrungen bei der Behandlung eines 73-jährigen männlichen Patienten mit histologisch gesichertem Basaliom der Schläfe mit Imiquimod.

\section{Kasuistik}

\section{Anamnese und Befund}

Ein 73-jähriger männlicher Patient wurde im April 2000 in unsere Klinik zur Therapie eines Basaliomrezidivs an der Nasenspitze eingewiesen. Seit Frühjahr 1999 bemerkte der Patient ein erneutes knotiges Wachstum im Bereich der Nasenspitze. Nebenbefundlich fielen zwei weitere klinisch suspekte Areale im Bereich der Schläfenregion links und am proximalen dorsalen Oberarm rechts auf. Beide, nur gering erhabenen und maximal $1,2 \mathrm{~cm}$ durchmessenden Herde, zeigten eine gerötete und schuppende Oberfläche mit im Seitenlicht prominentem Randsaum. Stanzbiopsien aus diesen Herden zeigten jeweils superfizielle Basaliome vom Typ des superfiziellen Basalioms (sog. Rumpfhautbasaliom) mit subepidermaler Coriuminfiltration, mittelgradiger Begleitentzündung und, im Falle des Tumors an der Schläfe, eine randliche Ulzeration mit Fibrin-Leukozytenschorf. Bis auf eine medikamentös eingestellte arterielle Hypertonie bestanden bei dem Patienten keine Begleiterkrankungen; im Routinelabor fanden sich keine Auffälligkeiten.

\section{Therapie und Verlauf}

Das Rezidivbasaliom an der Nase und das Basaliom am Oberarm wurden jeweils mikrographisch kontrolliert exzidiert. Betreffend das Basaliom an der Schläfe (Abb.1) schlugen wir dem Patienten vor, dieses im Rahmen eines individuellen Heilversuchs mit $5 \%$ Imiquimod-Creme (Aldara ${ }^{\circledR} 5 \%$ Creme, 3 M Medica, 46322 Borken) zu behandeln. Nach Aufklärung über die Behandlungsalternativen, Erfolgsaussichten und möglichen Nebenwirkungen willigte der Patient hierin schriftlich dokumentiert ein. Wir wiesen ihn an, 3-mal wöchentlich (Mo, Mi, Fr) die Creme auf das Areal aufzutragen. Nach einwöchiger Anwendungsdauer wurde der Patient in die ambulante Weiterbehandlung entlassen. Zu diesem Zeitpunkt fand sich im Bereich der Schläfe keine klinisch nennenswerte entzündliche Reaktion. Nach 4-wöchiger Behandlung stellte sich der Patient erneut vor. Es fand sich im Bereich der linken Schläfe ein ca. $5,5 \mathrm{~cm}$ durchmessendes deutlich infiltriertes und gering überwärmtes Erythem mit zentraler gelblich-grauer Krustenaufla- 


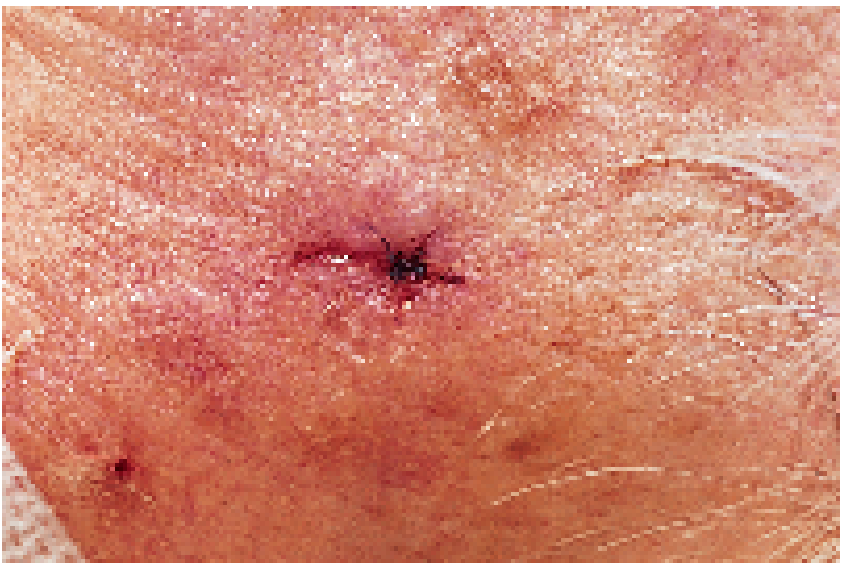

Abb. $1 \quad 1,2 \mathrm{~cm} \times 0,8 \mathrm{~cm}$ durchmessendes superfizielles Basaliom vom Rumpfhauttyp an der Schläfe eines 73-jährigen Patienten. Zustand unmittelbar nach diagnostischer Stanzbiopsie.

gerung (Abb. 2). Der ursprüngliche Tumor war in dem Areal klinisch nicht mehr auszumachen. Trotz der heftigen lokalen Entzündungsreaktion gab der Patient keine, beispielsweise grippeartigen, Allgemeinsymptome an. Auch die lokale Entzündungsreaktion selbst bezeichnete er als gut tolerabel. Bei einer weiteren Vorstellung nach 8-wöchiger Behandlungsdauer fand sich die lokale Entzündungsreaktion spontan deutlich rückläufig, es imponierten vor allem noch Krustenauflagerungen. Wir wiesen den Patienten an, die Behandlung bis zu einer Gesamtdauer von 12 Wochen bei gleicher Anwendungsfrequenz fortzusetzen. Drei Wochen nach Absetzen der Imiquimod-Therapie ohne spezielle Nachbehandlung stellte sich der Patient erneut vor. Es fanden sich keine Entzündungszeichen und klinisch sichtbaren Tumorreste mehr. Es bestand lediglich eine gering eingesunkene und hyperpigmentierte ca. $0,2 \mathrm{~cm}$ durchmessende Narbe im Bereich der vor Therapiebeginn entnommenen 4-mm-Stanzbiopsie (Abb.3). Eine zu diesem Zeitpunkt erneut durchgeführte 4-mm-Stanzbiopsie aus der unmittelbaren Umgebung zeigte keine Tumorzellen mehr, sondern eine unauffällige Epidermis. Im oberen Corium fanden sich, neben einer ungleichmäßig betonten aktinischen Elastose, fokal dichte chronisch-entzündliche Infiltrate. Da sich klinisch und histologisch keine Anhalte für einen Tumorrest fanden, bewerteten wir das Therapieergebnis als komplette Remission.

\section{Besprechung}

Die prinzipielle Wirksamkeit der lokalen Immuntherapie von Basaliomen (Basalzellkarzinomen) ist durch erfolgreiche Interferon-Behandlungen dieses Tumors nachgewiesen. Bei intratumoraler Injektion von 3-mal wöchentlich 1,5 Mio. IE Interferon alpha-2b bzw. 1,0 Mio. IE Interferon beta-1a über jeweils drei Wochen wurden bei insgesamt über 400 behandelten Patienten etwa $66 \%$ komplette Remissionen beobachtet $[2,3]$. Häufig geht eine lokale Entzündungsreaktion der Abheilung voraus. Als ein möglicher Wirkmechanismus der Interferontherapie wurde die Hemmung der Produktion des immunsuppressiven Zytokins IL-10 durch die Basaliomzellen nachgewiesen [5].

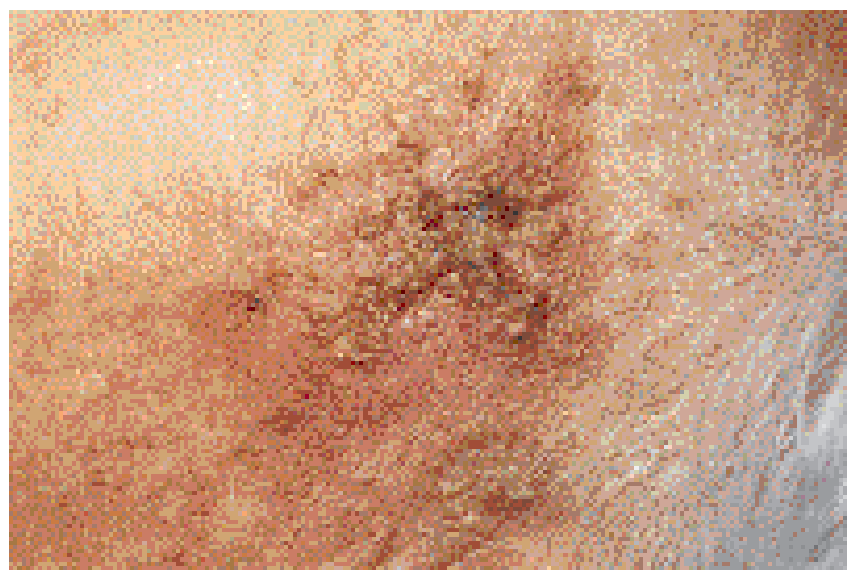

Abb.2 Zustand nach 4-wöchiger topischer Therapie mit 5\% Imiquimod-Creme 3-mal wöchentlich: Starke lokale Entzündungsreaktion von ca. $5,5 \mathrm{~cm}$ Durchmesser mit deutlicher Infiltration und Krustenbildung.

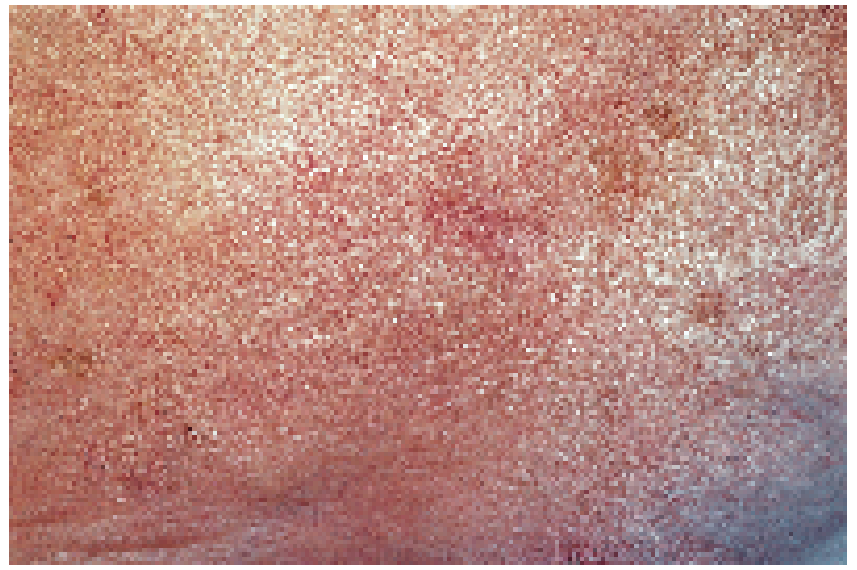

Abb. 3 Zustand 3 Wochen nach Ende der 12-wöchigen Behandlungsphase mit 5\% Imiquimod-Creme: Klinisch keine erkennbaren Basaliomreste oder Entzündungszeichen. Kleine Narbe als Residuum der diagnostischen Biopsie. Eine anschließend am Rand der Narbe durchgeführte Verlaufsbiopsie ergab auch histologisch keinen Hinweis auf verbliebene Tumorzellen.

Imiquimod ist ein topisch wirksamer Immunmodulator, der zur Therapie von Condylomata acuminata eingesetzt wird. Hierbei wird die Substanz als 5\% Creme 3-mal wöchentlich über maximal 16 Wochen angewendet [1]. Als Wirkungsmechanismen werden u. a. die Induktion der die TH1-Zell-Immunität fördernden Zytokine Interferon- $\alpha,-\beta$ und $-\gamma$ sowie IL-12 angenommen [6,7]. Kürzlich wurde eine Pilotstudie zur topischen Anwendung von 5\% Imiquimod-Creme bei zumeist superfiziellen Basaliomen von bis $\mathrm{zu} 2 \mathrm{~cm}^{2}$ Fläche publiziert [4]. Bei dreimal wöchentlicher Anwendung über im Mittel 14,5 Wochen wurde bei allen so behandelten 4 Patienten eine histologisch gesicherte komplette Abheilung erzielt. Weitere 7 Patienten, die täglich 2-mal 5\% Imiquimod-Creme über im Mittel 10 Wochen sowie 4 Patienten, die das Präparat 1-mal täglich für im Mittel 13 Wochen anwendeten, zeigten gleichfalls sämtlich eine komplette Abheilung. Geringere Anwendungshäufigkeiten und wirkstofffreie Behandlung mit der Grundlage waren auch bei 16 Wochen langer Therapiedauer 
weniger wirksam bzw. unwirksam. Bei 92\% der mit 5\% Imiquimod-Creme behandelten Patienten traten entzündliche Reaktionen im Bereich der topischen Anwendung des Präparates, systemische Nebenwirkungen (z. B. Nausea) bei bis zu $57 \%$ auf.

In unseren Händen kam es unter dem bei der Behandlung von spitzen Kondylomen bewährten Therapieschema mit 5\% Imiquimod-Creme 3-mal wöchentlich über insgesamt 12 Wochen zu einer histologisch verifizierten Abheilung eines superfiziellen Basalioms von ursprünglich ca. $1 \mathrm{~cm}^{2}$ Flächenausdehnung. Das kosmetische Ergebnis war ausgezeichnet. Die lokalen Nebenwirkungen waren ausgeprägt und ähnelten in ihrem Ausmaß und ihrer Dauer denen unter einer topischen Chemotherapie mittels 5-Fluor-Uracil (Efudix ${ }^{\circledR}$ ). Sie waren jedoch selbstlimitierend und klangen trotz fortlaufender Therapie wieder ab. Systemische Nebenwirkungen traten nicht auf; solche wurden auch in der Pilotstudie erst bei mindestens täglicher Anwendung des Präparates gesehen. Die mikrographisch kontrollierte Chirurgie ist bezüglich der niedrigen Rezidivrate von ca. $1 \%$ nach 5 Jahren [8] Therapiestandard beim Basaliom. Die Rezidivrate bei einer erfolgreichen Immuntherapie des Basalioms nach erfolgreicher Therapie mittels intraläsionalen Interferon $\beta$-Injektionen liegt nach im Mittel 2,4-jähriger Nachbeobachtungsdauer bei knapp 5\% [9] und somit in der Größenordnung der nach einfacher Schnittexzision, Elektrokaustik, Röntgen- oder Kryotherapie. Dagegen liegt die Rezidivrate nach topischer Chemotherapie z. B. mittels 5-Fluor-Uracil über 20\% [8]. Weitere umfangreichere Studien mit einem festen Behandlungsschema einschließlich mehrjähriger Nachbeobachtung sind zu fordern, ehe die Wertigkeit der topischen Immuntherapie mit Imiquimod beim Basaliom endgültig beurteilt werden kann. Interessant könnte diese experimentelle Behandlungsform beispielsweise für ausgewählte Patienten mit multiplen Basaliomen z.B. bei Gorlin-Goltz- bzw. Basalzell-Nävus-Syndrom sein [10]. Gegenwärtig kann die Anwendung von Imiquimod beim Basaliom in der Routine jedoch nicht empfohlen werden.

\section{Danksagung}

Wir danken Dres. med. Borodko und Lenz vom Institut für Pathologie und Tumordiagnostik des Vogtland-Klinikums Plauen (Komm. Chefarzt: Dr. med. Oertel) herzlich für die histologische Begutachtung der Biopsien und 3M Medica, Borken, für die Zurverfügungstellung der Imiquimod-Creme.

\section{Literatur}

${ }^{1}$ Beutner KR, Tyring SK, Trofatter KR, Douglas JM, Spruance SL, Owens ML, et al. Imiquimod, a patient applied immune response modifier for treatment of external genital warts. Antimicrob Agents Chemother 1998; 42: 789-794

2 Kowalzick L. Interferontherapie des Basalioms. In: Garbe C, Dummer R, Kaufmann R, Tilgen W (Hrsg.). Dermatologische Onkologie. Berlin, Heidelberg, New York: Springer, 1997: 171 - 181

${ }^{3}$ Kowalzick L, Rogozinski Th, Wimheuer P, Pilz J, Scholz A, Manske U, Fierlbeck G, Mohr P, Ochsendorf F, Wagner G, Müller R, Luger TA. Intratumorale Therapie des Basalzellkarzinoms (BCC) mit Interferon beta-1a: Ergebnisse bei 133 Patienten. Ztschr Hautkr 2000; 75: 301

${ }^{4}$ Beutner KR, Geisse JK, Helman D, Fox TL, Ginkel A, Owens ML. Therapeutic response of basal cell carcinoma to the immune response modifier imiquimod 5\% cream. J Am Acad Dermatol 1999; 41: $1002-1007$

${ }^{5}$ Kim J, Modlin RL, Dubinett SM, McHugh T, Nickoloff BJ. IL-10 production in cutaneous basal and squamous cell carcinomas: a mechanism for evading the local T cell immune response. J Immunol 1995; 155: 2240-2247

${ }^{6}$ Testerman TL, Gerster JF, Imbertson LM, Reiter MJ, Miller RL, Gibson SJ, et al. Cytokine induction by the immunomodulators imiquimod and S-27609. J Leukoc Biol 1995; 58: 365-372

7 Slade HB, Owens ML, Tomai MA, Miller RL. Imiquimod 5\% cream (Aldara). Exp Opin Invest Drugs 1998; 7: 437-439

${ }^{8}$ Rowe DE, Carroll RJ, Day CL. Long term recurrence rates in previously untreated (primary) basal cell carcinoma: Implications for patient follow-up. J Dermatol Surg Oncol 1989; 15: 315-328

${ }^{9}$ Kowalzick L, Rogozinski Th, Wimheuer P, Pilz J, Scholz A, Manske U, Fierlbeck G, Mohr P, Ochsendorf F, Wagner G, Müller R, Luger TA. Rezidivrate nach intratumoraler Therapie des Basalzellkarzinoms (BCC) mit Interferon beta-1a: Ergebnisse von 89 Patienten. Ztschr Hautkr 2000; 75: VIII

${ }^{10}$ Kagy MK, Amonette A. The use of imiquimod 5\% cream for the treatment of superficial basal cell carcinoma in a basal cell nevus patient. J Dermatol Surg 2000; 26: 577-578

\section{PD Dr. L. Kowalzick}

Klinik für Hautkrankheiten und Allergologie Vogtland-Klinikum Plauen $\mathrm{GmbH}$ Maximilian-Kolbe-Weg 1a 08529 Plauen 